\title{
Relacion entre autoconcepto físico, ansiedad y personalidad manifestada en usuarios de gimnasios Relationship between physical self-concept, anxiety and personality in gym clients *Ma Luisa Zagalaz Sánchez, **Rosario Castro López, ***Pedro Valdivia Moral, *Javier Cachón Zagalaz *Universidad de Jaén (España), **Universidad de Córdoba (España), ***Universidad de Granada (España)
}

Resumen. El propósito de este trabajo es estudiar el autoconcepto físico a través de la relación que posee con variables psicológicas como la ansiedad y la personalidad de usuarios de gimnasios. En el estudio han participado 154 sujetos, en edades comprendidas entre los 16 y 49 años (edad media de

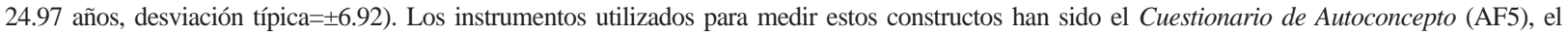
Cuestionario de Ansiedad Estado-Rasgo (STAI), y el Instrumento de Evaluación de la Personalidad (NEO-FFI). Los análisis de correlación efectuados han puesto de manifiesto la conexión entre las variables objeto de estudio entre las que destacan la relación inversa entre autoconcepto físico y ansiedad-rasgo. Por otra parte, en el estudio de regresión lineal realizado se han detectado las dimensiones Neuroticismo y Responsabilidad como aquellas que tienen mayor relación con el Autoconcepto físico.

Palabras clave. Autoconcepto físico, ansiedad, personalidad, musculación.

Abstract. The purpose of this work is to study physical self-concept through its relationship with psychological variables, such as anxiety and personality, in gym clients. The sample was composed by 154 participants aged between 16 and 49 years (mean age 24.97 years, SD = \pm 6.92 ). The instruments used to measure these constructs were the Self-Concept Questionnaire (AF5), the State Trait Anxiety Inventory (STAI), and the NEO FiveFactor Inventory (NEO-FFI). Correlation analyses confirmed significant connections among the variables studied, with emphasis on the inverse relationship between physical self-concept and trait anxiety. Moreover, linear regression analysis demonstrated that Neuroticism and Responsibility are the dimensions with strongest relation with physical self-concept.

Key words. Physical self-concept, anxiety, personality, workout.

\section{Introducción}

Entendemos el autoconcepto físico como la parte del general en la que se recogen todas las informaciones referidas al propio cuerpo (Ries, 2011) que proceden, tanto de la percepción subjetiva de las capacidades físicas (fuerza, flexibilidad, resistencia y velocidad) resumidas en el conjunto de condición física, como del atractivo físico (actitud hacia el propio cuerpo).

En lo que se refiere a la relación entre el autoconcepto físico y actividad física (AF) que abordamos en el presente estudio, existen numerosas investigaciones, desde bastante tiempo atrás, que han analizado las repercusiones o los beneficios que aporta el ejercicio físico, estableciéndose una importante relación con el mantenimiento de una buena salud mental y con la conformación de un autoconcepto positivo (Arruza, Arribas, Gil, Irazusta, Romero, \& Cecchini, 2008; García, Marín, \& Bohórquez, 2012; Castro, Molero, Cachón, \& Zagalaz, 2014; Reigal-Garrido, Videra-García, Parra, \& Juárez, 2012; Videra-García \& Reigal-Garrido, 2013; Louise, Hernández, Reigal, \& Morales, 2016; Reynaga-Estrada, Arévalo, Verdesoto, Jiménez, Preciado, \& Morales, 2016).

Con anterioridad, Jordan (1966), en Ayora, García-Ferriol, \& Rubio-Swift (1997), explica que la pronta obtención de éxito en las experiencias deAF, contribuye significativamente a mejorar el autoconcepto, la actitud y el estilo de vida de los estudiantes. Estos autores se habían apoyado en las ideas de Biles (1968), Samuelson (1969) y Dowell (1970) cuyas observaciones en las clases de educación física (EF) con escolares les habían hecho comprobar los cambios positivos en el autoconcepto, la experimentación del éxito con sentimientos de autovalía, e incluso una correlación positiva del autoconcepto y la capacidad física entre colegiales.

Más tarde, Schumacher, Small, \& Wood (1986) miden los efectos de la participación atlética sobre el autoconcepto y el rendimiento académico. La diferencia no fue significativa estadísticamente, no encontrándose tampoco disparidades cuando los grupos se separaron por sexos. Sin embargo, todas las puntuaciones de autoconcepto de los atletas fueron bastante más altas que las de los no atletas, puntuando más los varones deportistas. Por tanto la participación atlética mejora el autoconcepto de los adolescentes, con lo que coinciden con autores

Fecha recepción: 12-04-16. Fecha de aceptación: 19-11-16 Rosario Castro López

rosario.castro@unir.net anteriores (Dowell, 1970) y posteriores (Candel, Olmedilla, \& Blas, 2008).

En estudios posteriores, como se ha podido comprobar en la extensa revisión bibliográfica realizada, se ha considerado conveniente atender a tres cuestiones fundamentales, personalidad-autoconcepto, género y edad, que se desarrollan a continuación.

Primera, ¿̇en qué medida se benefician los jóvenes de la AF deportiva y qué efectos tiene sobre el desarrollo de la personalidad?, o ¿cuál es el grado de influencia que se establece entre ambas realidades -AF deportiva/personalidad-?, ya es cierto que en la conformación del autoconcepto parecen intervenir multitud de factores, entre ellos la práctica o no de una determinada AF (Esnaola, 2003; Godoy, Becker, Godoy, Vaccari, \& Michelli, 2001).

También es verdad que cuando se trata de aspectos de la salud física y social, la AF deportiva juega un papel importante en el fomento de la salud de los adolescentes. Numerosos estudios relacionan claramente los efectos beneficiosos del ejercicio físico sobre algunas de las dimensiones que conforman el autoconcepto y, particularmente, sobre el autoconcepto físico general (Knapen, Van-Coppenolle, Peuskens, Pieters, \& Knapen, 2006; Aróstegi, Goñi, Zubillaga, \& Infante, 2012). Esta cuestión fue investigada por Harter (1993) al encontrar en múltiples trabajos que las correlaciones entre la apariencia física y la autoestima eran asombrosamente altas y sólidas a lo largo de la vida, mucho más que ante cualquier otro tipo de variables como el rendimiento o la conducta, llevándole a preguntarse si la autoestima no sería cuestión de apariencia física.

Esnaola (2005) y Fox \& Corbin (1989) afirman que cualquier tipo de ejercicio físico practicado con regularidad tiene una influencia positiva sobre la percepción de la habilidad deportiva y condición física. Otros estudios manifiestan que la práctica habitual de AF deportiva puede ir unida a un estilo de vida más saludable, que los jóvenes se sienten menos agobiados y que se reducen síntomas como depresión, miedo, estrés o trastornos de alimentación (Calfas \& Taylor, 1994; Moreno, Cervelló, \& Moreno, 2008; Smith \& Biddle, 2008, Castro, Cachón, Valdivia, \& Zagalaz, 2015).

En un trabajo realizado con estudiantes deportistas en USA, Welk, Corbin, \& Lewis (1995) descubrieron que tenían una mejor percepción corporal que los deportivamente inactivos. Por lo que, según Brettschneider \& Heim (1997), el deporte a nivel de rendimiento puede abrir la posibilidad de contactar mejor con deportistas de la misma edad así como fomentar las facultades intelectuales. Whitehead \& Corbin (1997) llegan a la conclusión de que el deporte puede ser de gran utilidad 
en el desarrollo de la autoestima, siendo los niños con baja autoestima los más beneficiados. Richman (2002) es quien revela que las jóvenes adolescentes que han practicado de manera temprana algún tipo de actividad deportiva desarrollan una capacidad física y sensaciones más favorables sobre la valoración de su imagen corporal. En esa misma línea de trabajo, Drummond (2003) también llega a la conclusión, tras comparar dos grupos de deportistas que diferían en el nivel de experiencia, de que aquellos que destacan en AF presentan una identidad más positiva de su cuerpo, mientras que los menos expertos desarrollan un concepto pobre de su imagen.

Asimismo, en un trabajo realizado con 1203 sujetos de Sevilla sobre el por qué de la práctica físico-deportiva de la población, Gavala (2010) halló que las mujeres sevillanas, al igual que las de distintas poblaciones estudiadas, suelen practicar AF en mayor proporción como medio para mejorar su imagen corporal, o aumentar sus relaciones sociales, por lo que la relación de autoestima-autoconcepto físico se resalta mediante la práctica de ejercicio.

Si nos centramos únicamente en las subdimensiones que componen el autoconcepto físico y su relación con la práctica deportiva se han encontrado los estudios de Esnaola (2005) y Fox \& Corbin (1989), los cuales demostraron que la práctica deportiva habitual se relaciona positivamente con las subdimensiones de habilidad deportiva y condición física. Hayes, Crocker, \& Kowalski (1995) hallaron que únicamente la subescala de condición física mantenía relación con la AF en las mujeres, mientras que en los hombres todas las subescalas o subdimensiones correlacionaban con la práctica de AF. En la misma línea, Marsh \& Sonströem (1995) verificaron que la escala de condición física aparece más relacionada que ninguna otra del autoconcepto físico con la práctica de AF en mujeres. Moreno \& Cervelló (2005) comprobaron que los adolescentes que practicaban AF más de tres veces a la semana obtenían puntuaciones más altas en competencia deportiva, condición física, fuerza y atractivo físico que el resto. Goñi, Ruiz de Azúa, \& Rodríguez (2004) «en un estudio con adolescentes» pusieron de manifiesto que aquellos que realizaban deporte tenían puntuaciones más altas en todos los subdominios del autoconcepto físico, pero especialmente en los de habilidad deportiva y condición física. Estas puntuaciones fueron más altas cuanto más deporte realizaban los adolescentes, en lo que Gutiérrez, Sicilia, \& Moreno (1999) encontraron también, que aquellos universitarios que practicaban algún deporte obtenían mejores puntuaciones en todas las escalas del cuestionario.

Nuestra segunda cuestión va dirigida al género, ¿afecta por igual la práctica deportiva al desarrollo del autoconcepto físico a hombres que a mujeres? A este respecto, los estudios orientados al análisis de las diferencias de género muestran discrepancias entre ambos tanto para la dimensión global como para la física (Cachón, Cuervo, Zagalaz, \& González, 2015; Amezcua \& Pichardo, 2000; Asçi, Eklund, Whitehead, Kirazci, \& Koca, 2005; Eklund, Whitehead, \& Welk, 1997; Hagger, Biddle, \& Wang, 2005; Klomsten, Skaalvik, \& Espnes, 2004; Maïano, Ninot, \& Bilard, 2004; Pastor, Balaguer, \& García-Merita, 2003; Welk \& Eklund, 2005; Molero, Zagalaz, \& Cachón, 2013, Hortigüela, Pérez, \& Calderón, 2016). En este sentido, Molero, Ortega, Valiente, \& Zagalaz (2010) «en un estudio comparativo del autoconcepto físico en adolescentes» obtuvieron diferencias significativas a nivel estadístico en las percepciones realizadas en función del género en las escalas habilidad física, condición física, fuerza y autoconcepto físico general $(p<.05)$, a favor de los hombres. Por su parte Moreno, Cervelló, \& Moreno (2008) en su estudio indicaron una mayor autoestima, imagen corporal, competencia y condición física en los varones que las mujeres.

La tercera variable tomada en consideración de forma recurrente al estudiar las diferencias en el autoconcepto, es la relación entre autoconcepto físico y AF en función de la edad, es decir, ¿influye de igual manera la relación entre autoconcepto físico y AF en función de la edad del sujeto? En un meta-análisis realizado por Wilgenbusch \& Merrell (1999) sobre 22 estudios, combinando sexo y edad se advierte que existen diferencias significativas.

A pesar de los estudios presentados y como contraposición a lo expuesto sobre los beneficios de la práctica de AF, también hemos encontrado otros que muestran el lado «no saludable» del ejercicio físico, ya que no siempre la AF genera de forma automática efectos beneficiosos para el desarrollo personal, un ejemplo de ello es el estudio de Fernández, Contreras, García, \& González (2010), en el que declaran que en las actividades físico-deportivas practicadas con el fin de mejorar el aspecto físico o dónde la estética es importante (andar, footing, musculación, actividades de expresión o aeróbicos) los practicantes y especialmente las chicas adolescentes presentan peores percepciones físicas (atractivo físico, autoconcepto físico y general) que el sexo masculino.

Asimismo, la excesiva práctica de AF en muchas ocasiones conlleva trastornos de la conducta alimentaria en los deportistas (Castro, Cachón, Molero, \& Zagalaz, 2013), puede llegar a provocar adicción a la AF (Andrade, García, Remicio, \& Villamil, 2012; Zarauz \& RuizJuan, 2013), y la inadecuada planificación del ejercicio físico llega a generar insatisfacción personal, más aún si hacemos referencia al autoconcepto físico general (Bakker, 1988; Castro, 2013). En esta línea, Ben-Shlomo \& Short (1986) precisan que para mejorar las actitudes positivas hacia el concepto de uno mismo no es tan importante la ejecución de una determinada actividad deportiva como las características del programa de entrenamiento, coincidiendo con el estudio de Hortigüela, Pérez, \& Calderón (2016), por lo que después de haber realizado este riguroso análisis sobre los efectos que la $\mathrm{AF}$ que aporta al autoconcepto, el debate queda abierto a su estudio.

Expuestas estas ideas, el objetivo planteado en el presente trabajo es determinar las relaciones entre los niveles de autoconcepto físico y las variables de ansiedad y personalidad en una muestra de usuarios de gimnasios, al tratarse de un colectivo especialmente vulnerable que puede desencadenar adicción a la AF y/o insatisfacción corporal. De la misma manera, se pretende evaluar el poder predictivo de cada variable en función del autoconcepto físico. Todo ello con el propósito de conocer estas características personales para la posible prevención y tratamiento educativo de esta problemática, contrarrestando así los efectos negativos de la práctica de AF sobre la salud psicológica del deportista.

\section{Método}

\section{Participantes}

Se ha tratado una muestra de $n=154$ usuarios de gimnasios, con una media de edad de 24.97 años ( $D T=6.92)$, los cuales dedican al entrenamiento una media de 9.25 horas semanales $(D T=5.46)$. El estudio se ha hecho con los deportistas de musculación de diferentes gimnasios de la zona sur de España.

\section{Instrumentos}

Para medir las percepciones del autoconcepto físico fue utilizado $\mathrm{el}$ Cuestionario Autoconcepto Forma 5-AF5 (García \& Musitu, 2001). Esta escala está constituida por cinco factores de primer orden, de los cuales extraemos la información que contempla únicamente el autoconcepto físico.

Para evaluar los niveles de ansiedad de la muestra fue utilizado el cuestionario de Ansiedad Estado-Rasgo (STAI) elaborado por Spielberger, Gorsuch, \& Lushene (1970). El STAI es una escala de 20 ítems que aporta información de dos dimensiones, ansiedad estado y ansiedad rasgo.

El cuestionario de Evaluación de la personalidad (NEO-FFI), elaborado por Costa \& McCrae (1999), ha servido para determinar los factores de la personalidad de los deportistas. Consta de 30 ítems agrupados para dar respuesta a cinco dimensiones de la personalidad: neuroticismo, extraversión, apertura, amabilidad y responsabilidad.

\section{Procedimiento}

La recogida de datos se ha realizado en gimnasios y centros de fitness, pidiendo autorización previa para que los deportistas pudieran participar en el estudio. Con posterioridad, se explicó a los usuarios de gimnasios que sería una participación anónima y voluntaria. Los cuestionarios han sido auto-administrados, aunque la investigadora princi- 
pal estuvo presente mientras eran cumplimentados para resolver posibles dudas, controlando así que se completaban adecuadamente. Se rellenaron en los centros deportivos, siendo la duración media de 30 minutos. Finalmente, la muestra productora de datos fue de $n=154$.

\section{Análisis de datos}

Se efectuaron pruebas de correlación lineal aplicando el estadístico de Pearson. Se hizo un análisis de regresión por pasos sucesivos, incluyendo las variables de ansiedad y personalidad como predictores de autoconcepto físico, admitiéndose como válidos valores a nivel $p<.001$. Los análisis expuestos se realizaron con el programa estadístico SPSS v. 18.

\section{Resultados}

La Tabla 1 recoge los coeficientes de correlación de Pearson entre la escala del autoconcepto físico y las variables de ansiedad. Los resultados muestran elevados índices de correlación entre las variables de ansiedad y la de autoconcepto físico. Destacamos la correlación inversa entre la variable ansiedad-rasgo (Ries, Castañeda, Campos, \& Del Castillo, 2012) y los valores de autoconcepto físico ( $r=-.292$, $\mathrm{p}<.01)$.

La Tabla 2 recoge los coeficientes de correlación de Pearson entre la escala del autoconcepto físico y las variables de personalidad. Los resultados muestran que el autoconcepto físico se encuentra estadísticamente relacionado con cuatro dimensiones de las cinco del instrumento. Especial interés muestra la correlación negativa entre autoconcepto físico y neuroticismo ( $\mathrm{r}=-.295, \mathrm{p}<.001)$. Así como la concomitancia con la dimensión amabilidad ( $\mathrm{r}=.261, \mathrm{p}<.001)$. También se encontraron correlaciones significativas directas entre los valores de autoconcepto físico y extraversión ( $\mathrm{r}=.215$, p<.001), así como entre autoconcepto físico con responsabilidad ( $\mathrm{r}=.248 \mathrm{p}<.001)$.

En último lugar, se realizó un análisis de regresión en el que se consideró como variable dependiente el autoconcepto físico. Las variables independientes fueron las de ansiedad y personalidad anteriormente analizadas (Tabla 3).

Elmodelo resultante está compuesto por las variables Neuroticismo (de manera negativa) y Responsabilidad, correlacionando ambas dimensiones .354 con la variable dependiente, y explicando un total del 12.6\% de la variabilidad de Autoconcepto Físico [F $(2,151)=10.84 ; p<.05]$

Tabla 1.

Correlaciones entre Autoconcepto Físico y Ansiedad

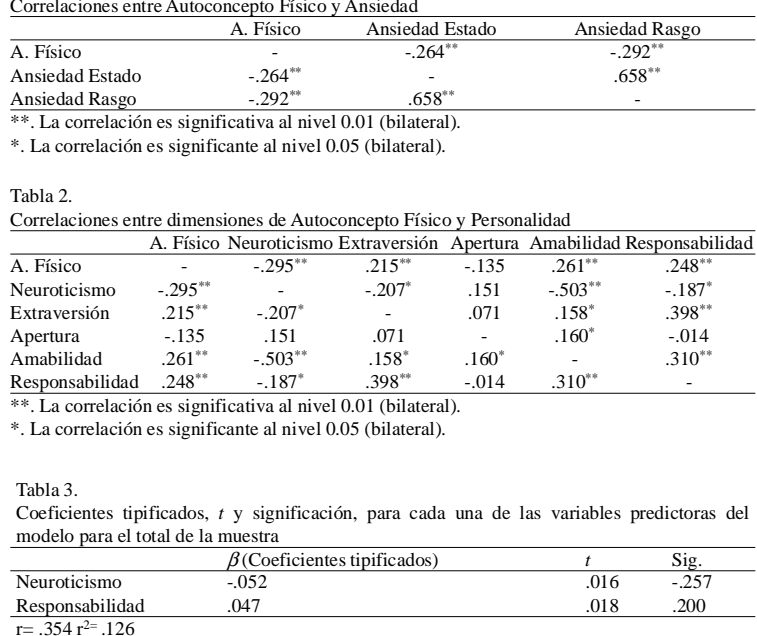

\section{Discusión}

La discusión pretende tomar dos enfoques diferentes en base a los resultados obtenidos en nuestro estudio: el primero busca contrastar la relación inversa entre autoconcepto físico y ansiedad en personas deportistas. Según Figueiras, González, \& Calvo (1996), la ansiedad es una de las variables que incide negativamente en el autoconcepto, ya que las personas que la padecen se encuentran sometidos con frecuencia a sentimientos de inutilidad produciendo en ellos una baja autoestima, con lo que las expectativas de rendimiento suelen ser bajas, reduciendo el esfuerzo realizado (Olmedilla, Ortega, \& Madrid, 2008; Mora \& Lobo-Di Palma, 2012). Esto coincide plenamente con los resultados de nuestro estudio ya que se obtuvo una relación estadística inversa entre ambos constructos. Siguiendo los resultados de los autores mencionados, a medida que aumenta la ansiedad, lo hace el autoconcepto negativo y disminuye el positivo, siendo esto más frecuente en las mujeres que en los varones. En esta línea, Newbegin \& Owens (1996) afirman que numerosas investigaciones han confirmado la relación inversa entre autoestima y ansiedad, destacando los estudios recientes propuestos por García-Más, Palou, Smith, Ponseti, Almeida, Lameiras, \& Leiva (2011), y Ríes, Castañeda, Campos, \& Del Castillo (2012).

Sobre las correspondencias entre las variables de la personalidad con el autoconcepto físico, Rhodes \& Smith (2006) y Hoyt, Rhodes, Hausenblas, \& Giacobbi (2009) afirman que la práctica deportiva se asocia positivamente con los rasgos de responsabilidad (p.ej., tendencia al orden, autodisciplina y orientación al logro), y extraversión (p. ej., sociabilidad, actividad, búsqueda de estimulación y afecto positivo) y, negativamente, con el rasgo neuroticismo (p.ej., inestabilidad emocional y vulnerabilidad a la de-presión). Los sujetos de nuestra muestran también exhiben la vinculación anunciada obteniendo resultados significativos, además de presentarla también con la dimensión amabilidad. Esta última podría ser vinculada al optimismo, constructo que ha sido estudiado por Ntoumanis, Taylor, \& Standage (2010) y así mismo está vinculado a niveles elevados de autoconcepto, en este caso de forma inversa ya que éstos afirman que los sujetos con miedo al fracaso tienden a utilizar en mayor medida la estrategia del pesimismo defensivo que, a su vez, correlaciona de forma negativa con el autoconcepto físico y el disfrute en la práctica deportiva.

\section{Conclusiones}

Analizados los datos y respondiendo al objetivo del estudio planteado, se extraen las siguientes conclusiones:

1. La ansiedad está relacionada de manera inversa con el autoconcepto físico.

2. Los niveles de personalidad también se relacionan con el autoconcepto físico, habiéndose obtenido relaciones inversas con la variable neuroticismo y directas con las variables responsabilidad, amabilidad, y extraversión.

\section{Referencias}

Amezcua, J. A. \& Pichardo, M. C. (2000). Diferencias de género en autoconcepto en sujetos adolescentes. Anales de Psicología, 16(2), 207-214.

Andrade, J. A., García, S., Remicio, C., \& Villamil, S. (2012). Niveles de adicción

al ejercicio corporal en personas fisioculturistas. Revista Iberoamericana del Ejercicio y el Deporte, 7(2), 209-226.

Aróstegi, B., Goñi, A., Zubillaga, A., \& Infante, G. (2012). El autoconcepto físico de jóvenes futbolistas de alto rendimiento. Cuadernos de Psicología del Deporte, 13(1), 9-14.

Arruza, J. A., Arribas, S., Gil De Montes, L., Irazusta, S., Romero, S., \& Cecchini, J.A. (2008). Repercusiones de la duración de la Actividad Físico-deportiva sobre el bienestar psicológico. Revista Internacional de Medicina y Ciencias de la Actividad Física y el Deporte, 8(30), 171-183.

Asçi, F., Eklund, R. C., Whitehead, J. R., Kirazci, S., \& Koca, C. (2005). Use of the CY-PSPP in other cultures: a preliminary investigation of its factorial validity for Turkish children and youth. Psychology of Sport and Exercise, 6, 33-50.

Ayora Pérez, D.; García Ferriol, A., \& Rubio Swift, S. (1997). Factores del autoconcepto relacionados con el rendimiento de los adolescentes en educación física. Revista de Psicología del Deporte, 12, 59- 
73.

Bakker, F. C. (1988). Personality differences between young dancers and non-dancers. Personality and individual differences, 9(1), 121131.

Ben-Shlomo, L. S. \& Short, M. A. (1986). The effects of physical conditioning on selected dimensions of self-concept in sedentary females. Occupational Therapy in Mental Health, 5, 27-46.

Biles, F. (1968). Self concept changes in college freshmen women in a basic physical education course using two methods of instruction. Tesis Doctoral. Ohio State University.

Brettschneider, W. \& Heim, R. (1997). Identity, sport and youth development. In K. R., Fox(Ed.), The physical self: From motivation to well-being (pp. 205-228). Leeds: Human Kinetics.

Cachón Zagalaz, J., Cuervo Tuero, C., Zagalaz Sánchez, M. L., \& González González de Mesa, C. (2015). Relación entre la práctica deportiva y las dimensiones del autoconcepto en función del género y la especialidad que cursan los estudiantes de los grados de magisterio. Journal of Sport and Health Research, 7(3), 257-266.

Calfas, C .J. \& Taylor, W. C. (1994). Effects of physical activity on psychological variables of children. Pediatric Exercise Science, 6, 406-423.

Candel, N., Olmedilla, A. \& Blas, A. (2008). Relación entre la práctica de actividad física y el autoconcepto, la ansiedad y la depresión en chicas adolescentes. Cuadernos de Psicología del Deporte, 8(1), 61-77.

Castro López, R. (2013). Diferencias de personalidad, autoconcepto, ansiedad y trastornos de alimentación en deportistas de musculación: patrones psicológicos asociados a la vigorexia. Tesis Doctoral: Universidad de Jaén.

Castro López, R., Cachón Zagalaz, J., Molero López-Barajas, D., \& Zagalaz Sánchez, M. L. (2013). La dismorfia muscular y su relación con los síntomas de trastornos de la conducta alimentaria. Revista Mexicana de Trastornos Alimentarios, 4(1), 31-36.

Castro López, R., Cachón Zagalaz, J., Valdivia Moral, P. Á., \& Zagalaz Sánchez, M. L. (2015). Estudio descriptivo de trastornos de la conducta alimentaria y autoconcepto en usuarios de gimnasios. Revista iberoamericana de psicología del ejercicio yel deporte, 10 (2), 251-258.

Castro López, R., Molero, D., Cachón, J., \& Zagalaz Sánchez, M. L. (2014). Factores de la personalidad y fisicoculturismo: indicadores asociados a la vigorexia. Revista de Psicología del deporte, 23(2), 295-300.

Costa, P. T. \& McCrae, R. R. (1999). NEO-PI-R. Inventario de Personalidad Neo Revisado (NEO-PI-R). Madrid: TEA Ediciones.

Dowell, L. J. (1970). A study of the relationship between selected physical attributes and self-concepts. In G. Kenyon (Ed.), Contemporary Psychology of Sport (pp. 657- 672). Chicago: The Athletic Institute.

Drummond, M .J. N. (2003). The meaning of boys' bodies in physical education. The Journal of Men's Studies, 11(2), 131-143.

Eklund, R. C., Whitehead, J. R., \& Welk, G. J. (1997). Validity of the children and youth physical selfperceptions profile: a Confirmatory Factor analysis. Research Quarterly for Exercise and Sport, 68, 249-256.

Esnaola, I. (2003). Autoconcepto físico y práctica deportiva. In F.V., Castro, Ma. I., Fajardo, M. I. Ruiz, y A., Ventura, (Eds.), Contextos psicológicos de aprendizaje (pp.167-179). Fuerteventura: PSICOEX.

Esnaola, I. (2005). Elaboración y validación del cuestionario AutoKontzeptu Fisikoaren Itaunketa (AFI) de autoconcepto físico. Tesis Doctoral. Universidad del País Vasco.

Fernández, J., Contreras, O., García, L. M., \& González Villora, S. (2010). Autoconcepto físico según la actividad físico-deportiva realizada y la motivación hacia ésta. Revista Latinoamericana de Psicología, 42(2), 251-263.

Figueiras, M. E., González, A., \& Calvo, J. (1996). Ansiedad en el ámbito educativo. Un estudio de su relación con variables de perso- nalidad y autoconcepto. Actas al I Congreso de la SEAS. Alicante.

Fox, K. R. \& Corbin, C. B. (1989). The Physical Self-Perception Profile: Development and preliminary validation. Journal of Sport and Exercise Psychology, 11, 408-433.

García, A. J., Marín, M., \& Bohórquez, M. R. (2012). Autoestima como variable psicosocial predictora de la actividad física en personas mayores. Revista de Psicología del Deporte, 21(1), 195-200.

García, F. \& Musitu, G. (2001). Autoconcepto Forma 5. AF5. Manual. Madrid: TEA.

García-Más, A., Palou, P., Smith, R. E., Ponseti, X., Almeida, P., Lameiras, J., \& Leiva, A. (2011). Ansiedad competitiva y clima motivacional en jóvenes futbolistas de competición, en relación con las habilidades y el rendimiento percibido por sus entrenadores. Revista de Psicología del Deporte, 20(1), 197-207.

Gavala, J. (2010). Practical reasons of physical activity of the people of Sevilla. Journal of Sport and Health Research. 3(3),169-178.

Godoy, R. F., Becker, B. B., Godoy, D. V., Vaccari, P., \& Michelli, V. (2001). El efecto del ejercicio sobre los niveles de ansiedad, depresión y autoconcepto de pacientes con dolencia pulmonar obstructiva crónica. Revista de Psicología del Deporte, 10(2), 267-278.

Goñi, A., Ruiz deAzúa, S., \& Rodríguez Fernández, A. (2004). Deporte y autoconcepto físico en la preadolescencia. Apunts, Educación Física y Deportes, 77, 18-24.

Gutiérrez, M., Sicilia, A., \& Moreno, J.A. (1999). Autoconcepto físico y práctica deportiva de una muestra de estudiantes universitarios. En Actas del IV Congreso de las Ciencias del Deporte, la Educación Física y la Recreación (pp. 199-213). Lleida: INEFC.

Hagger, M. S., Biddle, S. J. H., \& Wang, C. K. J. (2005). Physical selfperceptions in adolescence: Generalizability of a multidimensional, hierarchical model across gender and grade. Educational and Psychological Measurement, 65, 297-322.

Harter, S. (1993). Causes and consequences of low self-esteem in children and adolescents. In R. B., Baumeister(Ed.), Self-esteem: The puzzle of low self-esteem (pp. 57-78). Nueva York: Plenum Press.

Hayes, S. D., Crocker, P. R., \& Kowalski, K. (1995). Gender differences in physical self-perceptions, global self-esteem and physical activity: evaluation of Physical Self-Perception Profile model. Journal of Sport Behavior, 22(1), 1-14.

Hoyt, A., Rhodes, R., Hausenblas, H., \& Giacobbi, P.(2009). Integrating five- factor model facet-level traits with the theory of planned behavior and exercise. Psychology of Sport and Exercise, 10, 565572.

Hortigüela Alcalá, D., Pérez Pueyo, A., \& Calderón Luquín,A. (2016). Efecto del modelo de enseñanza sobre el autoconcepto físico del alumnado en educación física. Retos, Nuevas tendencias en Educación Física, Deporte y Recreación, 30, 76-81

Klomsten, A. T., Skaalvik, E. M., \& Espnes, G A. (2004). Physical selfconcept and sports: do gender differences still exist? Sex Roles, 50, 119-127.

Knapen, J., Van-Coppenolle, J., Peuskens, G, Pieters, G., \& Knapen, K. (2006). Comparison of changes in physical fitness, physical self-concept, global self-esteem, depression and anxiety following two different psicomotor therapy programs in non-psychotic psychiatric impatients. In A. Prescott (Ed.), The concept of self in education, family and sports (pp. 91-114). New York: Nova Science Publishers.

Louise Kyle, T., Hernández Mendo, A., Reigal Garrido, R. E., \& Morales Sánchez, V. (2016). Efectos de la actividad física en el autoconcepto y la autoeficacia en preadolescentes, Retos, Nuevas tendencias en Educación Física, Deporte y Recreación, 29, 61-65.

Maïano, C., Ninot, G., \& Bilard, J. (2004). Age and gender effects on global self-esteem and physical selfperception in adolescents. European Physical Education Review, 10, 53-69.

Marsh, H. W. \& Sonstroem, R. J. (1995). Importance ratings and specific components of physical self-concept: relevance to predicting global components of self-concept and exercise. Journal of Sports and Exercise Psychology, 17, 84-104.. 
Molero, D., Ortega, F., Valiente, I., \& Zagalaz, M. L. (2010). Estudio comparativo del autoconcepto físico en adolescentes en función del género y del nivel de actividad físico-deportiva. Retos, Nuevas tendencias en Educación Física, Deporte y Recreación, 17, 38-41.

Molero, D., Zagalaz, M. L., \& Cachón, J. (2013). Estudio comparativo del autoconcepto físico a lo largo del ciclo vital. Revista de Psicología del Deporte, 22(1), 135-142.

Mora, A. L. \& Lobo Di Palma, J. J. (2012). Autoestima y Ansiedad Social en Personas con Discapacidad Visual que Participan en Deporte Competitivo y en Actividades Recreativas. Des-encuentros, 9, 44-54.

Moreno, J.A. \& Cervelló, E. (2005). Physical self-perception in spanish adolescents: effects of gender and involvent in physical activity. Journal of Human Movement Studies, 48, 291-311.

Moreno, J. A., Cervelló, E., \& Moreno, R. (2008). Importancia de la práctica físico-deportiva y del género en el autoconcepto físico de los 9 a los 23 años. International Journal of Clinical and Health Psychology, 8(1), 171-183.

Newbegin, I. \& Owens, A. (1996). Self-esteem and anxiety in secondary school achievement. Journal of Social Behavior and Personality.

Ntoumanis, N. \& Taylor, M. (2010). Testing a model of antecedents and consequences of defensive pessimism and self-handicapping in school physical education. Journal of Sport Sciences, 28, 15151525.

Olmedilla, A., Ortega-Toro, E., \& Madrid, J. (2008). Variables sociodemográficas, ejercicio físico, ansiedad y depresión en mujeres: un estudio correlacional. Revista Internacional de Medicina y Ciencias de la Actividad Física y el Deporte, 8(31), 224-243.

Pastor, Y., Balaguer, I., Pons, D., \& García-Merita, M. L. (2003). Testing direct and indirect effects of sports participation on perceived health in Spanish adolescents between 15-18 years of age. Journal of Adolescence, 26, 717-730.

Reigal-Garrido, R., Videra-García, A., Parra Flores, J. L., \& Juárez Ruiz de Mier, R. (2012). Actividad físico deportiva, autoconcepto físico y bienestar psicológico en la adolescencia. Retos: Nuevas tendencias en Educación Física, Deporte y Recreación, 22, 19-23.

Reynaga-Estrada, P., Arévalo Vázquez, E. I., Verdesoto Gáleas, A. M., Jiménez Ortega, I. M., Preciado Serrano, M. L., \& Morales Acosta, J. J. (2016). Beneficios psicológicos de la actividad física en el trabajo de un centro educativo. Retos, nuevas tendencias en Educación Física, Deportey Recreación, 30, 203-206.

Rhodes, R. E. \& Smith, N. E. I. (2006). Personality correlates of physical activity: a review and meta-analysis. British Journal of sports medicine, 40(12), 958-965.
Richman, S. E. (2002). Relative foraging value to lesser scaup ducks of native and exotic clams from San Francisco Bay. Tesis Doctoral. University of Wyoming.

Ries, F. (2011). El autoconcepto físico en adolescentes sevillanos en función del sexo y de la evolución de la carrera deportiva. Retos, Nuevas tendencias en Educación Física, Deportey Recreación, 19, 38-42.

Ries, F., Castañeda, C., Campos, M. C., \& Del Castillo, O. (2012). Relación entre ansiedad-rasgo y ansiedad-estado en competiciones deportivas. Cuadernos de Psicología del Deporte, 12(2), 9-16.

Samuelson, G. F. (1969). The effects of a specially structured seven week physical education class upon the self-concept of low-esteem tenth grade girls. Tesis Doctoral. University of North Carolina. Greensboro.

Schumacher, J. F., Small, L., \& Wood, J.(1986). Self-concept, academic achievement, athletic participation. Perceptual and Motor Skills, 62, 387-390

Smith,A. L. \& Biddle, S. J.H. (Eds.) (2008). Youth Physical Activity and Sedentary Behavior - Challenges and Solutions. Champaign, IL.:Human Kinetics.

Spielberger, C. D., Gorsuch, R. L. y Lushene, R. E. (1970). Manual for the State-TraitAnxiety Inventory. PaloAlto: Consulting Psychologist Press.

Videra-García, A. \& Reigal-Garrido, R. (2013). Autoconcepto físico, percepción de salud y satisfacción vital en una muestra de adolescentes. Anales de Psicología, 29(1), 141-147.

Welk, G. J. \& Eklund, B. (2005). Validation of the children and youth physical self perceptions profile for young children. Psychology of Sport and Exercise, 6, 51-65.

Welk, G. J., Corbin, C., \& Lewis, L. (1995). Physical self-perceptions of high school athletes. Pediatric Exercise Science, 7, 152-161.

Whitehead, J. R. \& Corbin, C. B. (1997). Self-Esteem in Children and Youth: The role of Sport and Physical Education. In K. R. Fox (Ed.), The Physical Self (pp. 175-203). Champaign, IL: Human Kinetics.

Wilgenbusch, T. \& Merrell, K. W. (1999). Gender Differences in SelfConcept among Children and Adolescents: A Meta-Analysis of Multidimensional Studies. School Psychology Quaterly, 14(2), 101120.

Zarauz Sancho, A. \& Ruiz-Juan, F. (2013). Variables predictoras de la adicción al entrenamiento en atletas veteranos españoles. Retos: Nuevas tendencias en Educación Física, Deporte y Recreación, 24, 33-35.

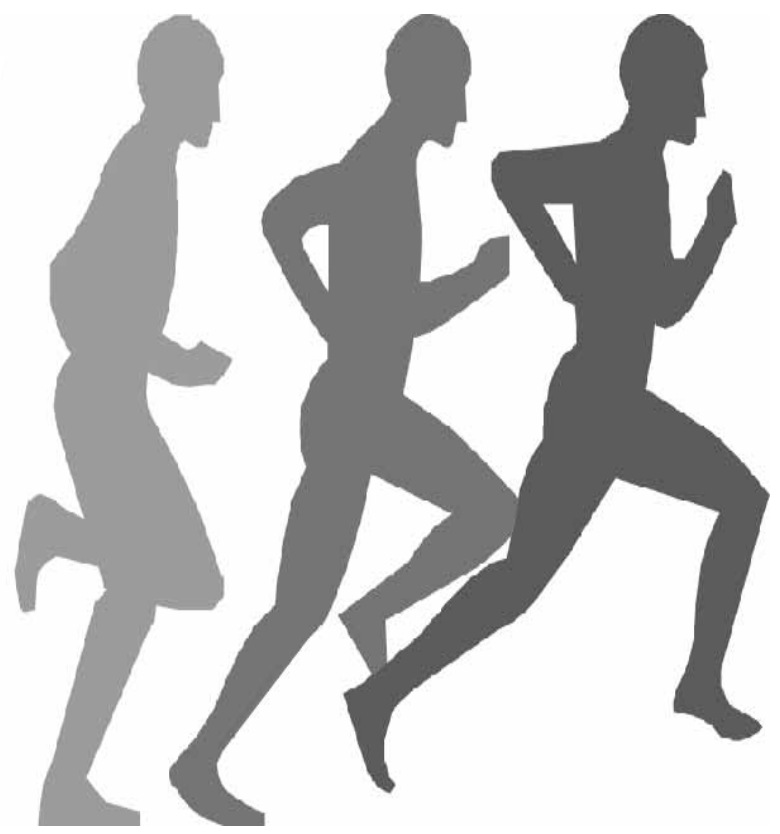

